Article

\title{
Les vicissitudes de l'intégration de la dimension spatiale dans la pensée économique
}

\begin{abstract}
Akli Akerkar ${ }^{1}$ 亦
1 Montpellier SupAgro, France

Résume. Depuis l'antiquité les auteurs englobaient dans leurs réflexions la dimension spatiale de l'économie. Mais dès le début du XIXe siècle jusqu'aux années 1950, période correspondant à la prédominance du paradigme néoclassique, l'espace occupe une place marginale et ambiguë dans la pensée économique. Cela s'explique d'une part, par le paradigme de la rationalité parfaite sur lequel s'est constituée l'économie politique classique et son approche hypothéticodéductive et, d'autre part, par des facteurs géographiques, historiques et intellectuels des pays ainsi que du degré de développement de leur système de transport. Il fallait attendre le retournement des hiérarchies spatiales des années 1970 pour voir dans la notion d'espace celle du territoire, c'est-à-dire un milieu socio-spatial structurant des comportements spatiaux, de l'organisation industrielle et de l'innovation.

Mots-clés: Economie a-spatiale, théories de localisation, croissance régionale, développement régional, économie territoriale.
\end{abstract}

\begin{abstract}
Since ancient times, researchers have included in their reflections the spatial dimension of the economy. But from the beginning of the 19th century until the 1950s, a period corresponding to the predominance of the neoclassical paradigm, space occupied a marginal and ambiguous place in economic thought. This is explained on the one hand, by the paradigm of perfect rationality on which the classical political economy and its hypothetical-deductive approach was built up and, on the other hand, by geographic, historical and intellectual factors of the countries as well as the degree of development of their transport system. We had to wait for the reversal of the spatial hierarchies of the 1970s to see in the concept of space that of the territory, that is to say a socio-spatial environment structuring spatial behavior, industrial organization and innovation.
\end{abstract}

Keywords: A-spatial economy, location theories, regional growth, regional development, territorial economy.

\section{CORRESPONDENCE:}

2677 avenue Etienne Méhul, 34070 Montpellier.

@akerkarakli@gmail.com 


\section{Introduction}

Situer dans le temps le début de réflexion sur l'espace n'est pas une tache évidente. Néanmoins, on sait que durant l'antiquité, la pensée économique fait des relations ville/campagne la base de l'organisation de l'espace et qu'au Moyen Age, la justice spatiale est au centre des préoccupations des auteurs. Elle est souhaitable entre les régions, les royaumes et seigneuries et entre villes et campagnes (Dockès, 1969).

En étudiant la mobilité des hommes, des capitaux et des entreprises, les auteurs préclassiques du XVIe et XVIIIe siècles cherchaient, quant à eux, à comprendre les processus conduisant à la formation des équilibres et des déséquilibres spatiaux. Ils intègrent dans leurs analyses trois types de circuits possibles : le circuit international, le circuit interrégional et le circuit ville/campagne. L'apparition de déséquilibre ou de l'équilibre spatiale dépend de rapports (coopération/domination) qu'entretiennent les différents points d'un circuit donné.

Le XIXe siècle marque une période de déclin de l'intérêt qu'accordent les économistes aux problématiques spatiales. En effet, du début du XIXe siècle jusqu'aux années 1950, période correspondant à la prédominance du paradigme néoclassique dans la science économique, l'espace occupe une place marginale et ambiguë dans la pensée économique. Cette négligence donne l'impression que les activités économiques se déroulent sur une tête d'épingle (Combes, Mayer et Thisse, 2006) et que l'économie est une sorte de "wonderland of no spatial dimensions " (Isard, 1956, pp. 25-26).

En parallèle à ce marginalisme économique en vogue dans le monde anglosaxon, se développe en Allemagne un ensemble de courants de pensée qui rejettent les thèses a-spatiales néoclassiques et dont les travaux portent essentiellement sur les problématiques de l'organisation de l'espace. Cependant, Si les travaux des cameralistes sont réputés pour avoir tenté de structurer économiquement les territoires allemands durant le XVIIe et le XVIIIe siècles, et ceux des spatialistes allemands pour avoir jeté les bases de l'analyse spatiales durant le XIXe et le XXe siècles, leur impact demeure largement interne et méconnu à l'étranger.

Ce mouvement est vite relayé à partir du début des années 1950 par les travaux de l'école américaine, la Regional Science Association (RSA) et dans les années 1960 par les différents courants de la Science Régionale de l'Europe occidentale, entre autres l'Association de Science Régionale de Langue Française (ASRDLF). La Science régionale est perçue en Europe, en URSS et aux USA comme un outil permettant un aménagement du territoire sur des bases scientifiques (Bailly, 2009).

Le retournement des hiérarchies spatiales durant les années 1970 a apporté une approche différente dans l'analyse des dynamiques spatiales. La capacité d'un milieu territorial à endogénéiser son développement grâce aux jeux combinant marché et 
proximité devient l'hypothèse principale des différents courants scientifiques qui s'intéressent désormais aux problématiques du développement régional.

C'est dans cette dialectique de la perception de l'espace que s'inscrit cet article en cherchant d'une part, à comprendre les raisons de son ignorance puis de son intégration lente et tardive dans la pensée économique et d'autre part, à analyser les différentes manières dont il est appréhendé depuis les préclassique à nos jours.

\section{L’espace dans la pensée économique préclassique}

Les théories économiques spatiales modernes ne sont pas de création ex nihilo, leurs fondements s'appuient sur des travaux qui leur sont antérieurs et qui ne cessent de les alimenter et de les enrichir constamment. A ce propos Dockès (1969, p. 424) écrit "La théorie spatiale d'aujourd'hui et celle des XVIIe et XVIIIe siècles se développement autour de concepts voisins ».

Pour les préclassiques, l'équilibre spatiale est atteint si les relations entre les différents endroits du circuit sont symétriques et complémentaires, c'est-à-dire les flux d'hommes, de produits, de capitaux, d'entreprises, etc. se réalisent en direction de tous les sens. En conséquence, l'interdépendance et l'interaction des différents points conduits à leur croissance simultanée. A contrario, un circuit spatial est déséquilibré, si les rapports entre les différents points du circuit sont disproportionnés et asymétriques, c'est-à-dire les flux de biens, de capitaux et $\mathrm{d}^{\prime}$ hommes s'orientent seulement en direction du centre au détriment du reste de l'aire. L'influence asymétrique fait que la croissance du centre résulte non pas de la coopération mais de la domination et de l'exploitation de la périphérie par le centre.

Durant cette période où prédominent les pensées mercantiliste et physiocratique, les réalités de l'espace économique sont analysées en termes de moyens et de voies de communication, de courants commerciaux et des centres de négoce, de la répartition des villes et de la localisation des implantations industrielles, de l'occupation du sol dans l'agriculture, d'obstacles à la mobilité des hommes, des marchandises et des capitaux, etc.

Sans prétendre à l'exhaustivité nous nous limitons à présenter certaines conceptions majeures de l'espace qui prévalaient à une époque de très grandes réflexions qui préfigurent l'avènement de l'économie politique au sens sous lequel elle est connue aujourd'hui.

L'une des figures les plus marquantes de cette époque est Bodin. Ces nombreux et divers travaux constituent sans doute un substrat riche et solide sur lequel seront développées plus tard, respectivement la théorie de la spécialisation internationale, les théories de la formation d'agglomérations, la théorie quantitative de la monnaie et les nouvelles théories de l'émigration. Pour faire allusion à la nécessité de la division internationale du travail, il compare le fonctionnement du monde à celui du corps 
humain. Les différentes régions du monde, comme les organes du corps humain, ont chacune une fonction particulière dont l'intégration par les échanges culturels et commerciaux est indispensable pour leur épanouissement. Aucun pays ne peut subvenir seul à ses propres besoins et donc la politique autarcique est néfaste. A ce propos il écrit ${ }^{2}$ "Quant à la traite des marchandises qui sortent de ce royaume, il y a plusieurs grands personnages qui s'efforcent, et se sont efforcés de la retrancher du tout, s'il leur été possible: croyant que nous pouvons vivre heureusement et à grand marché sans rien bailler ni recevoir de l'étranger, et ne saurions nous en passer » (Bodin, 1568/1593, p. 59).

Si Bodin préconise le libre échange, il est convaincu que ce dernier est plus profitable pour certaines nations que d'autres «qu'il n'y a personne qui gagne qu'un autre n'y perde » (Bodin, 1576/1593, p. 933)³. Ainsi, pour protéger l'économie nationale contre la concurrence étrangère, il recommande l'instauration d'une politique protectionniste: "Quant aux matières qu'on apporte des pays étrangers, il est besoin de rabaisser l'impôt, et de le hausser aux ouvrages de main, et ne permettre qu'il en soit apporté de payé étranger» (Bodin, 1576/1593, p. 877)4. Les fondements de la richesse d'une nation reposent sur l'abondance des terres fertiles, des métaux précieux et surtout d'hommes. Pour éviter leur hémorragie, Bodin cherche les moyens de contrôler leurs mouvements et souligne les avantages énormes d'une politique d'immigration avantageuse pour les étrangers qu'il considère comme source de capitaux et de fortune.

Très influencé par la philosophie libérale de Hobbes et convaincu de la supériorité des lois naturelles sur les lois positives. Petty, est partisan d'une inégale répartition de richesses entre les nations, les régions, les hommes, la ville et la campagne, la capitale et les provinces, etc. Pour lui, ce sont ces inégalités spatiales qui conduisent à l'échange et à la prospérité, elles paraissent donc légitimes et naturelles. Une telle organisation favorise d'une part; la division du travail qui permet une production abondante, moins coûteuse et de meilleure qualité : "Dans une grande cité, il sera aisé de diviser le travail [...], les industries s'y engendreront l'une l'autre, et chaque industrie sera divisée en autant de parties que possible, afin que le travail de chaque artisan soit simple et facile $»^{5}$ (Petty, 1905, p. 521). Et d'autre part, l'avènement de la concurrence qui pousse à la baisse des prix et à l'amélioration de la qualité "nous voyons de même que dans les villes et dans les rues d'une grande ville où tous les habitants font le même commerce, le produit spéciale à ces endroits est mieux fait et moins coûteux qu'ailleurs $»^{6}$ (Petty, 1905, p. 521). En étudiant le cas de Londres et le rôle capital de River Thames (Tamise) sur son expansion, il montre le rôle important des voies de

\footnotetext{
2 Idée reprise par P. Dockès (1969 : 87).

3 Idée reprise par P. Dockès $(1969: 90)$.

4 Idée reprise par P. Dockès (1969: 90).

5 Idée reprise par P. Dockès (1969: 138).

6Idées reprise par P. Dockès (1969: 139).
} 
communications et des moyens de transport sur l'apparition et le développement des villes. Les avantages d'une grande ville réside dans sa capacité à raccourcir les circuits économiques en concentrant en un lieu peu étendu les populations, les artisans, les marchands, etc.

Si la concentration urbaine occupe la grande partie des travaux de Petty, l'espace rural n'est pas exclu de ses analyses. Plus d'un siècle avant von Thünen, il étudie les rapports entre villes et campagnes, en s'intéressant principalement à la localisation des activités agricoles et à la formation de la rente foncière. Il schématise le lien entre la ville et la rente comme suit : l'accroissement de la demande agricole de la ville provoque la hausse des prix des denrées alimentaires qui engendre à son tour l'accroissement de la rente dont dépend le prix de la terre.

Quelques années plus tard, les travaux de Petty inspirent ceux de Cantillon. En revanche, ces deux auteurs, s'opposent radicalement sur la perception de l'organisation de l'espace et surtout sur les relations entre villes et campagnes. Si Petty est partisan des inégalités spatiales et de la concentration, Cantillon prône la justice spatiale et la dispersion.

Dans «Essai sur la nature du commerce en général» (1755/1997), Cantillon donne une vue d'ensemble de la vie économique en étudiant la répartition des populations et de leurs activités ainsi que les relations interrégionales. Pour lui, la structuration de l'espace résulte de la contrainte des coûts de transport croissants avec la distance, d'où l'emboîtement hiérarchique des villages, des bourgs, des villes et de la capitale. Le groupement d'un ensemble d'aires de dimension inférieure donne naissance à une aire de dimension supérieure. En effet, le village naît de la nécessité des cultivateurs de vivre tout près de leurs terres. Le besoin de s'approvisionner en outils et d'écouler leurs denrées fait naître un marché où plusieurs petits entrepreneurs, marchants et artisans sont encouragés à s'y établir, le village s'agrandit pour se transformer ainsi en bourg. Ce dernier devient à son tour une ville après s'y installer les seigneurs et les grands propriétaires terriens.

Les relations ville-campagne sont structurellement déséquilibrées en faveur de la première. Le développement de la première dépend de l'appauvrissement de la seconde. Ce déséquilibre spatial résultant de l'échange inégal, s'exprime en termes des prix de la terre, du travail et des coûts de transport pénalisant la campagne. La cause en est la grande inégalité de répartition des terres qui conduit les grands propriétaires terriens à s'installer dans les villes et de ce fait à l'allongement des circuits commerciaux. Leurs gros revenus favorisent l'apparition de manufactures qui à leur tour attirent de plus en plus de nouveaux capitaux. Ces flux à sens unique créent une situation défavorable aux campagnes et rendent les inégalités permanentes auxquelles Cantillon souhaite y remédier. Il plaide pour ce qu'on appelle aujourd'hui la convergence régionale; en élaborant une théorie de la décentralisation et de dissémination industrielle, qui permet une relocalisation et 
l'installation des manufactures près des sources de matières premières et dans des provinces; et en préconisant le retour sur leurs terres des riches fonciers. Par ces recommandations, Cantillon entend favoriser la consommation locale et le raccourcissement des circuits commerciaux pour réduire la dette des campagnes visà-vis des villes.

Cependant, si Cantillon se distingue par sa plaidoirie pour ce que l'on nomme une politique volontariste visant une justice spatiale, il reste fidèle à l'idée des auteurs libéraux du XIIIe siècle considérant les déséquilibres interrégionaux comme naturels et inévitables. "...si le prince fait la distribution des terres par portions égales à tous les habitants, elles ne laisseront pas dans la suite de tomber en partage à un petit nombre: Un habitant aura plusieurs enfants, et ne pourra laisser à chacun d'eux une portion de terre égale à la sienne... » (Cantillon, 1755/1997, p. 3).

Environ un siècle après les travaux de Petty et une décennie après ceux de Cantillon, les Principles de Steuart (1767/1789) ont fait progresser et enrichi la théorie de l'espace économique. A plusieurs égards, les analyses de Steuart semblent être imprégnées par celles de ses prédécesseurs Petty et Cantillon. Cependant, entre ces deux positions diamétralement opposées, Steuart se veut un mercantiliste modéré et cherche à trouver un compromis entre la concentration inévitable chère au premier et la dispersion souhaitée par le second. A son tour, il étudie la localisation des manufactures, des hommes et des activités agricoles, l'apparition et la croissance des agglomérations, les relations entre les villes et les campagnes immédiatement avoisinantes et lointaines.

L'apport majeur de Steuart réside dans son analyse originale et atypique de l'apparition des villes. Contrairement à Bodin pour qui, à défaut de terres fertiles dans les régions difficiles, les hommes sont contraints de s'adonner à d'autres activités autre que l'agriculture, ce qui conduit progressivement à l'érection d'une cité. Steuart soutient que, plus les terres sont productives, moins est le nombre d'hommes nécessaires pour les cultiver et plus sont importantes et nombreuses les villes. On comprend bien donc, que l'apparition des villes et leur extension n'étaient plus dues comme il a été souvent affirmé, à la stérilité des terres et à la localisation de propriétaires fonciers et des rentiers, mais plutôt, à la performance des campagnes qui, en dégageant un surplus d'hommes et de subsistances, facilitent l'installation de manufactures et par conséquent la croissance de villes nouvelles. Le marché d'une manufacture contrairement à celui d'un artisan est le plus souvent national, ainsi, sa localisation n'est plus déterminée par la proximité du marché urbain mais plutôt par la proximité à la fois des sources d'énergies et des matières premières, des lieux où les prix des vivres sont bas et enfin, des lignes de transport et de communication. Soucieuses de leurs approvisionnement, les manufactures s'érigent aux «bords des rivières et des ruisseaux, quand on emploie des machines qui exigent de l'eau; au voisinage des forêts, lorsqu'on a besoin de feu; au lieu qui fourni la matière de la manufacture, comme 
les différentes mines de métaux, de charbon, les briques, etc. » (Steuart, 1767/1789, pp. 8788).

Steuart perçoit les rapports ville-campagne en termes d'interdépendance et de renforcement mutuel. Contrairement à ce que suppose Cantillon, les déséquilibres régionaux sont conjoncturels et passagers, et la concentration ne présente pas que des inconvénients. Les grandes agglomérations sont bénéfiques aussi bien pour les citadins que pour les ruraux. En effet, si l'agrandissement des villes résulte de l'exode rural, l'accroissement des populations urbaines engendre une demande supplémentaire en vivres, et la hausse des prix qui en résulte stimule les activités agricoles des campagnes.

Durant la seconde moitié du XVIIIe siècle, le déclin de l'agriculture française et la misère du monde rural attirent l'attention des physiocrates. Dans son tableau économique (1758/2005), présenté comme le précurseur des modèles économiques modernes, Quesnay interprète l'économie comme un circuit fermé. Partant d'un postulat que seule l'agriculture est productive, il analyse comment le produit net part de la classe productive et y revient après le passage par la classe des propriétaires puis de celle-ci à la classe stipendiée.

Tout comme Petty, le souci majeur des physiocrates est d'abréger les circuits. Mais si pour Petty la solution réside dans la concentration des industries, des énergies, des hommes et d'autres richesses dans les villes, les physiocrates qui sont sans doute notablement influencés par Cantillon, mettent l'accent sur la renaissance des campagnes, le danger de l'accumulation des fortunes (destructives) dans les villes et une répartition plus juste des richesses dans l'espace. Pour eux, les grandes villes nuisent aux campagnes, leur accroissement qui devrait résulter d'un enrichissement préalable des provinces, se fait par l'appauvrissement de ces dernières. En guise d'illustration, Quesnay fait allusion à Rome : «C'était à la vérité les impositions excessives sur les provinces qui soutenaient le luxe dans Rome. Mais lorsque les provinces furent ruinées, cette grande ville ne pût se soutenir par elle-même, ni conserver sa domination » (Quesnay, 1757-8/2005, p. 285) et d'ajouter «jamais Rome ne fut si riche et si somptueuse que lorsqu'elle dévastait les provinces qu'elle avait assujetties à sa domination; mais sa splendeur était la flamme de l'incendie qui consommait les forces de l'empire et qui le soumit à la puissance de ses ennemis » (Quesnay, 1757-8/2005, p. 315). Les villes privent les campagnes de leurs facteurs essentiels de développement que sont les capitaux et les hommes, il s'en suit une déprise et une pauvreté qui poussent les populations à l'exode rural: "Quand les campagnes sont maltraitées, appauvries, sans sûreté pour la culture des biens, et sans ressource pour les hommes qui les habitent, ils se réfugient et s'accumulent dans les grande villes » (Quesnay, 1757-8/2005, p. 311). Pour renverser les tendances et améliorer le sort des provinces, les physiocrates recommandent un reflux de capitaux vers les campagnes, mettent en avant les avantages de la 
consommation locale, encourage l'industrie rurale et incitent au retour des propriétaires sur leurs terres.

En résumé, les auteurs préclassiques ont jeté les bases de la théorie économique spatiale, en posant en des termes modernes les problèmes inhérents à la localisation des hommes et des activités et les rapports ville/campagne. Toutefois, ce courant de pensée alliant analyse économique et analyse spatiale disparaît avec la vogue du marginalisme et le triomphe de la microéconomie au XIXe siècle. Il faut attendre le XXe siècle pour que l'analyse spatiale se développe au tour de l'économie spatiale, régionale et urbaine et plus récemment de l'économie territoriale.

\section{Le triomphe de la pensée économique marginaliste au XIXe siècle et le déclin de l'analyse spatiale}

Les travaux de ce courant de pensée à dominante anglo-saxonne s'inscrivaient dans une économie ponctiforme (Ponsard, 1955). Les fondateurs de la microéconomie traditionnelle et de la théorie moderne de l'équilibre général, principalement Hicks et Samuelson raisonnaient comme si les processus de production et de consommation se déroulaient en un seul point. «The latest contributors have concentrated their efforts on attacking the problem of time to exclusion of that of space. Hicks, Mosak, Lange, and Samuelson, to name a few, have all treated an economy in which all factors and producers, commodities and consumers are, in effect, congregated at one point " (Isard, 1956, p. 25).

Il est fort légitime de s'interroger sur les raisons qui font que l'espace occupe une place subsidiaire chez les économistes depuis le début du XIXe siècle et pendant un siècle et demi. Une autre interrogation qui découle de la première est de savoir pourquoi l'intégration de l'espace dans l'analyse économique est si lente et incohérente? Si l'on sait que depuis Ibn Khaldoun à Smith (Aydalot, 1985), les chercheurs englobaient dans leurs réflexions la dimension spatiale de l'économie et que jusqu'à la fin du XIIIe siècle la séparation entre économie spatiale et économie ponctiforme n'est pas encore accomplie (Dockès, 1969).

\subsection{Le paradigme de la rationalité parfaite, une erreur épistémologique}

L'ignorance des dimensions spatiales par les scientifiques notamment les économistes classiques et néoclassiques au profit des considérations temporelles est justifiée par le fait que, la formation et l'étude de plusieurs phénomènes économiques nécessitent peu ou prou la prise en compte de l'espace (Thisse, 2007). Selon A. Marshall, le coût de production n'a aucune influence perceptible sur le cours de certains produits sur le marché, ceci tient "aux différences relatives à l'espace et à la

période de temps sur lesquelles s'étend le marché en question; l'influence du temps étant plus fondamentale que celle de l'espace » (Marshall, 1890/1906 : 168). 
Plusieurs auteurs (Dockès, 1969), (Ponsard, 1988a), (Aydalot, 1985), (Lepetit,1988) et (Thisse, 1997) sont unanimes pour voir dans l'abandon de l'espace, une rupture méthodologique entre les classiques et les préclassiques. L'économie préclassique est essentiellement inductive, contrairement à l'économie politique classique qui, prétendant au statut scientifique de l'économie, fait une grande place à l'abstraction et à une approche hypothético-déductive dont les lois ne sont valables que si l'on tient compte d'hypothèse implicites ou explicites (Dockès, 1969). Cette erreur épistémologique tient au paradigme de la rationalité parfaite sur lequel s'est constituée la science économique (Perrin, 1992).

En effet, avec le modèle d'économie politique pure de Walras (1952) et ses hypothèses de l'homo oeconomicus et de la concurrence pure et parfaite, la science économique se définit moins comme une science de la richesse que comme une science de l'action rationnelle. Dans cette perspective, les impacts et les conséquences des comportements sociaux, religieux, politiques, etc. sur la répartition de l'activité économique et la circulation des richesses disparaissent (De Lamarlière et Staszak, 2000).

Cette hypothèse est celle d'E. Durkheim qui, vers la fin du XIXe siècle porte un regard critique à l'égard de la manière de faire des économistes. Il critique les fondements méthodologiques de l'économie politique et sa vision réductrice à la fois de l'économie et de la société. En se voulant une science autonome et indépendante, l'économie politique nie l'encastrement de l'économie dans la société (Polanyi, 2009) en tentant de se séparer et de s'isoler des phénomènes sociaux. En considérant les évènements économiques comme des faits distincts par rapport aux interactions sociales, aux idées morales et aux règles du droit « le meilleur moyen de les étudier n'estil pas de faire abstraction de ces causes perturbatrices qui en altèrent l'évolution naturelle?» (Durkheim, 1887/1975, p. 274). Or pour E. Durkheim, ces « causes perturbatrices » réagissent assez souvent sur le cours des évènements économiques et le modifie. En procédant ainsi, les économistes isolent les actes économiques d'un « homme général et logique ", sans valeurs objectives parmi l'ensemble des actes de l'homme réel (l'homme vraiment homme) immergé dans le milieu qui l'entoure (Durkheim, 1887, p. 279). Ainsi, c'est tout l'objet de la science économique qui se voit remis en cause. Or, «qu'on le veuille ou non, qu'elles soient un bien ou un mal, les sociétés existent. C'est au sein de sociétés constituées que se manifeste l'activité économique. La logique ne peut rien contre un fait qui complique, il est vraie, les données du problème, mais dont il n'est pas possible de faire abstraction» (Durkheim, 1886/1970, p. 208). Pour Durkheim, ce processus d'abstraction conduit les économistes à l'hypothèse de l'homo oeconomicus qui n'est rien d'autre que la dénaturation de l'homme en tant qu'être social, en le dépouillant de ses caractéristiques historiques et sociales. «Pour simplifier les choses les économistes l'ont artificiellement appauvri. Non seulement ils ont fait abstraction de toutes les circonstances de temps et de lieux, de payer pour imaginer le type 
abstrait de l'homme en général, mais dans ce type idéal lui-même ils ont négligé tout ce qui ne se rapportait pas à la vie strictement individuelle, si bien que d'abstraction en abstraction il ne leur est plus resté en main que le triste portrait de l'égoïste en soi » (Durkheim, 1888, p. 85).

Dans la même lignée des critiques à l'égard des assises méthodologiques de l'économie orthodoxe dominante Aydalot (1985) évoque les raisons des réticences des néoclassiques à intégrer l'espace dans leurs modèles d'analyses. La définition d'un optimum économique qui leur est cher, passe par les mécanismes naturels du marché, c'est-à-dire par des ajustements prix/quantités qui rétroagissent en cas de déséquilibre pour retrouver un état d'équilibre stable. Toutefois cette situation n'est possible que si l'on suppose que les ajustements interviennent immédiatement et gratuitement, autrement dit, sans délais ni coûts. Que se passerait-il si on raisonnait dans une économie réelle où ces ajustements nécessitent un délai et supportent un coût ? La distance et les coûts entraînent un bouleversement du système d'équations du modèle et par conséquent tout équilibre est impensable. Ainsi, si les résultats des modèles néoclassiques sont valables pour une économie a-spatiale, leur généralisation à l'économie spatiale nécessite de dépasser certaines hypothèses et une adaptation au cadre d'analyse globale de cette dernière. Comme l'écrit Ponsard (1975 : I) «L'économie normative, comme toute la théorie économique générale, est construite à l'aide d'hypothèses qui évacuent, explicitement ou implicitement, l'espace ; en conséquence, ses résultats valent pour une économie réduite à un point et seulement pour une telle économie; ils ne sont généralement pas susceptibles d'être étendus au cas d'une économie spatiale. Toute tentative de prise en compte des facteurs spatiaux suppose leur introduction explicite dans le corps d'hypothèses, la pleine reconnaissance de leur spécificité et la parfaite adaptation du cadre de l'analyse ». Dès lors, on comprend mieux pourquoi l'économie abstraite a souvent nié et minimisé le rôle du facteur spatial et privilégié le facteur temporel. Les néoclassiques cherchent à évacuer et à éviter tout facteur susceptible de rendre l'équilibre inconcevable. Pour eux, l'espace est un gêneur, "il apparaît comme l'introduction du loup dans une bergerie, du concret dans une discipline qui se veut abstraite » (Aydalot, 1985, p. 10). Derycke (1994, p. 19) le qualifie comme « une véritable trouble-fête qui modifie radicalement les perspectives et les conclusions de l'analyse aspatiale ». A son tour Camagni (2004, p. 13) écrit «privilégier la dimension temporelle signifié pouvoir maintenir le raisonnement économique dans le cadre de variables purement économiques, observées dans leurs mouvements et leur évolution, tant dis que prendre en considération la dimension spatiale aurait «contaminé » la pureté de l'analyse économique avec des éléments de caractère social, culturel, institutionnel ».

\subsection{Les raisons objectives}

Bien que les raisons de la marginalisation de l'espace en théorie économique soient encore mal connues (Thisse, 1992), souvent peu satisfaisantes (Benko, 1998, 2008) et ne sont pas totalement claires (Derycke et Huriot, 1996), l'explication la plus 
répandue parmi les auteurs est liée à la baisse considérable à la fois des coûts de transport et du temps, conséquence directe de la révolution industrielle qui a conduit au raccourcissent des distances à un rythme croissant entre les lieux (Bairoch, 1997). Toutefois cette explication est à relativiser, l'effacement total des coûts de transport est inconcevable et leur existence aussi faible soit-elle continue à influencer les agents économiques quant à leurs calculs et décisions économiques.

L'explication de Jacobs (1984) reprise par Thisse (1997) nous fournit une idée éclairée de la question, dans la mesure où son raisonnement repose sur des faits et théories économiques dominants aux XVIIIe et XIXe siècles. Les fondateurs de l'économie classique fidèles à la vision mercantiliste de pays, considérés comme des entités dominantes permettant de comprendre la vie économique, conduit à la négligence des territoires infra- nationaux. Les nations seraient alors homogènes et la seule référence à l'espace est la frontière nationale.

L'importance ou non accordée à l'espace dépend aussi des facteurs géographique, historique et intellectuel des pays ainsi que du degré de développement de leur système de transport (Gérard-Varet et Thisse, 1997 ; Thisse, 1997 ; Combes, Mayer et Thisse, 2006). En Angleterre, l'intégration des marchés internes grâce au réseau de transport dense, et le faible coût des échanges maritimes insérant l'économie du pays dans un vaste empire colonial, amènent les économistes anglais à négliger les échanges infranationaux, minorer le rôle de la distance et à bâtir une théorie des échanges internationaux sans coût de transport. Cette approche s'est imposée depuis son apparition et ne cesse d'influencer la pensée économique moderne. En revanche, le processus lent de la formation de la nation allemande au XIXe siècle, l'unification et l'élargissement tardive de son marché interne, l'importance du transport terrestre et son coût élevé, le rôle primordial qu'occupe le concept de territoire dans la philosophie politique allemande, etc. constituent autant de raisons qui expliquent pourquoi l'espace a constamment retenu l'attention des économistes allemands. En effet, en Allemagne, contrairement aux autres pays occidentaux tels que la France et l'Angleterre, la théorie économique, s'est développée sous une forme spécifique, en plaçant dès sa naissance, au XVIIe siècle, l'espace au centre de ses réflexions. Pour Garner (2001), deux principales raisons font du territoire une catégorie centrale dans les sciences camérales. D'une part, aux XVIIe et XVIIIle siècle, comparativement à la France et l'Angleterre, l'Allemagne ne constitue pas un Etat-nation centralisé. L'empire est politiquement et économiquement morcelé. D'autre part, les conséquences dramatiques de la Guerre de Trente Ans, rend urgent un redressement économique et démographique des territoires. Pour ce faire, les gouvernements de ces derniers se voient accorder une reconnaissance de la supériorité territoriale (Landeshoheit), en les émancipant de la tutelle de l'empereur. 
Si les travaux des cameralistes sont réputés pour avoir tenté de structurer économiquement les territoires allemands durant le XVIIe et le XVIIIe siècles, et ceux des spatialistes allemands pour avoir jeté les bases de l'analyse spatiales ${ }^{7}$ durant le XIXe et le XXe siècles, leur impact demeure largement interne et méconnu à l'étranger. La raison en est que l'école historique allemande est sortie vaincue de la querelle des méthodes (Methodenstreit) qu'il a opposé à l'école autrichienne. Pour Nussbaumer (2002) en se basant sur les analyses de Hodgson (2001), l'école allemande n'a pu développer des arguments rigoureux sur la méthode empirique de l'historisme, ainsi, l'empirisme prônait par Schmoller était largement démenti par les arguments logiques de Menger. Sur les questions spatiales, l'école historique allemande procède par une approche pluridisciplinaire, se fondant sur le raisonnement inductif et multicausale pour comprendre le processus historique de formation des configurations spatiales des activités économiques. De son côté, l'école de Vienne ; fidèle aux paradigmes néoclassiques, adopte une démarche déductive plaidant pour l'isolation puis la compréhension des causes purement économiques de la localisation des activités économiques.

\section{La naissance de la science régionale, de l'espace au territoire}

Comme nous pouvons le constater, l'intégration aussi partielle soit-elle de l'espace à la théorie économique durant la première moitié du $X X^{e}$ siècle est presque exclusivement le fait de l'école spatialiste allemande.

Après plus d'un demi-siècle de son existence, l'économie régionale englobe un grand nombre d'approches, de théories et de modèles. De ce renouveau de l'intérêt des économistes pour l'espace (Bourdeau-Lepage et Huriot, 2009), deux réponses sont fournies à la question de la nature du processus de développement régional : le développement fonctionnel et le développement territorial. On y retiendra deux grandes orientations ou approches (Capello, 2009; Lacour, 2009) dans la façon dont l'espace est inséré dans les différents modèles théoriques élaborés jusqu'à nos jours. L'une relève de l'analyse spatiale pure et s'inscrit dans le prolongement de la tradition de l'analyse économique spatiale classique en ce qu'elle s'attache à la réactivation des théories de la localisation; elle est une focalisation sur la croissance régionale dans un espace uniforme et abstrait. L'autre montre une préoccupation croissante pour le développement régionale et l'aménagement du territoire, elle se veut une rupture avec la conception de l'espace fonctionnel pour privilégier l'espace relationnel diversifié « diversified-relational space » (Capello, 2009).

7Le bilan de l'histoire de l'analyse économique spatiale fait état de la formation de quatre grands modèles de référence que Ponsard (1988) qualifie de paradigmes autour desquels gravitent certains types de recherche. Ils sont par ordre chronologique du paradigme de von Thünen (1826), du paradigme de Weber (1909), du paradigme de Hotelling (1929) et enfin de celui de Christaller (1933) et de Lösch (1940). 
La première orientation a été adoptée par un large groupe de théories de la croissance régionale qui se sont développées à partir de diverses branches de l'économie dominante au début des années 1950 et 1960. Il s'agit entre autres de la théorie néoclassique du commerce interrégionale de Heckscher (1919) et Ohlin (1933), des modèles néoclassiques de la croissance régionale, notamment celui de Borts et Stein (1964), et de la théorie de la base exportatrice s'inspirant des théories keynésiennes de la croissance régionale de l'après-guerre de Hoyt (1954) et North (1955). Les travaux de ces différents courants restent dominés par une vision matérialiste du monde du fait de l'utilisation des concepts, des théories et des mesures de l'économie classique au détriment des approches des sciences sociales. Dans une telle démarche l'espace n'existe pas en tant que tel et n'a pas d'intérêts propres. Il apparaît comme un substrat pur des phénomènes économiques, basés seulement sur des distances et des lieux sans tenir compte des attributs et des caractéristiques du milieu. Il n'est donc pas plus qu'un contenant physique du développement qui joue un rôle purement passif dans les processus économiques.

Depuis le début des années 1990 ce courant a connu un profond renouvellement suite aux travaux de Krugman qui jettent les bases fondatrices de la "Nouvelle économie géographique ». Dans un article célèbre: "Increasing Returns and Economic Geography » (1991), il explique les raisons de concentration des activités dans une région en transposant les modèles des rendements croissants de la théorie du commerce international à la géographie économique. En réactivant ce qu'il appelle les cinq «traditions bannies » de la géographie économique : la théorie de localisation, la physique sociale, la causalité cumulative, la modélisation de l'utilisation du sol et les externalités locales marshallienne, il manifeste une forte inclinaison pour la théorie de la localisation (Martin et Sunley, 2000) et une certaine affiliation à la vielle Régional Science de W. Isard. Si on reconnait à Krugman de s'être parvenu à modéliser pour la première fois des résultats issus d'une longue tradition (Coissard, 2007), de vouloir « réintroduire la géographie dans l'analyse économique » (Martin et Sunley, 2000,p. 37) et de créer des modèles géographique basés sur la concurrence imparfaite (Benko, 1999b), les critiques qui lui sont adressées par les géographes et économistes, adeptes d'approches marxistes et régulationnistes, sont parfois virulentes. Pour Martin (1999), la formalisation mathématique sur laquelle s'appuie la démarche de Krugman au détriment d'une approche empirique, le conduit à négliger le monde réel. Dans le même ordre d'idée, Lipietz lui reproche de n'avoir analysé que les paramètres qu'il peut formaliser et son incapacité à expliquer et à mesurer les conséquences des externalités de l'offre (Benko, 1999a). De leur côté, Courlet et Pecqueur (2013, p. 34) tout en soulignant l'originalité de Krugman concernant la mise en évidence du développement régional comme processus historique dépendant des trajectoires antérieures suivies, constatent que pour des 
raisons méthodologiques, "tout ce qui est influence exercée par les structures institutionnelles, sociales et culturelles est délaissé ».

La seconde orientation, adoptée par les théories du développement régional, tente de revisiter la notion de l'espace et de reconsidérer son rôle. Dans les années 1950, elles regroupent un ensemble d'analyses qui remet en cause la théorie néo-classique de la convergence et met plutôt l'accent sur la croissance déséquilibrée ; il s'agit essentiellement de la théorie des pôles de croissance de Perroux et de la théorie de la causalité circulaire et cumulative de Myrdal. L'apport théorique de ces auteurs a ensuite contribué dans les années 1970 et 1980 à l'émergence de plusieurs courants de pensée constitutifs de «l'Economie territoriale » visant à expliquer le rôle actif de l'espace dans les modes d'organisation et la dynamique des phénomènes économiques.

L'approche de Perroux suppose que "la croissance est déséquilibrée. Le développement est déséquilibré. L'implantation d'un pôle de développement suscite une suite de déséquilibres économiques et sociaux »(Perroux, 1961/1969, p. 205). La propagation spatiale de la croissance est inégale et qu'elle est l'œuvre de pôles de croissance localisés, il théorise ainsi, le fait que la croissance économique a une dimension spatiale: "la croissance n'apparait pas partout à la fois; elle se manifeste en des points ou pôles de croissance, avec des intensités variables; elle se répand par divers canaux et avec des effets terminaux variables pour l'ensemble de l'économie »(Perroux, 1955, p. 309).

Un pôle de croissance est caractérisé par une ou plusieurs unité(s) dominante(s) et motrice (s) qui, en s'installant dans une région peu industrialisée, pourrait (aient) provoquer un effet de développement en chaîne et par conséquent, un effet de polarisation. Son (leur) action organisatrice et motrice, où s'allient trois composantes que sont la force contractuelle (pouvoir de négociation), la dimension et la nature d'activité (Perroux, 1948, 1950), va se sentir en aval comme en amont. Néanmoins, ces effets ne se propagent pas également au profit de tous les secteurs et sur l'ensemble du territoire mais se coagulent au voisinage des unités motrices. Ainsi, la théorie de pôle de croissance se présente à la fois comme théorie de croissance sectoriellement déséquilibrée et comme théorie de croissance régionale déséquilibrée.

Myrdal (1957/1959) ajoute un éclairage important en mettant en évidence le principe de causalité cumulative circulaire. Il montre que dans des systèmes interdépendants, les rétroactions positives (positive feedback) sont la règle, de sorte que le processus de polarisation tend de plus en plus à se renforcer. Les économies d'agglomération, les rendements d'échelle croissants, les imperfections du marché, les qualifications, les infrastructures, etc. conduisent à un processus cumulatif de différence de croissance entre régions, et engendrent un cercle vicieux qui maintient les régions en retard de développement en position dominée et les régions en avance en position dominante. Ce processus simultané de renforcement de la richesse ou de la pauvreté est provoqué par les effets de remous centripète «backwash effects » qui font que l'expansion d'une localité draine vers elle les mouvements de main- 
d'œuvre, de capitaux, de marchandises, de service et de commerce au détriment des autres régions. Myrdal n'exclut pas l'existence des effets de propagation centrifuge " spread effects » depuis les centres d'expansion qui, eux, profiteraient aux localités périphériques. Néanmoins, l'observation empirique de la polarisation de l'économie, fait dire à Myrdal que ce sont les effets de remous qui l'emportent finalement sur les effets de propagation.

Si ces approches présentent un changement conceptuel par rapport aux hypothèses néo-classiques et ont le mérite de préparer un terrain promoteur à revisiter la notion de l'espace et à reconsidérer son rôle au cœur des dynamiques localisées, elles demeurent prisonnières d'une conception réductrice de l'espace ; elles n'impliquent pas manifestement un abandon radical d'un espace support.

Vers le milieu des années 1970 on assistait au retournement des hiérarchies spatiales. Au moment où des régions d'une très grande tradition industrielle connaissaient des difficultés de croissance, des dynamismes économiques dans des «régions qui gagnent» (Benko et Lipietz, 1992) jadis périphériques voyaient le jour dans de nombreux pays à travers le monde. Ce sont ainsi les fondements théoriques des approches traditionnelles du développement régional qui se trouvaient démentis et remis en cause. Parallèlement, de nombreuses approches tentent de rendre compte de cette transformation de la problématique et des orientations du développement régional en mettant plutôt l'accent sur la notion de territoire, amorçant ainsi un renouvellement radical des analyses des dynamiques socio-économiques locales.

Toutes les études consacrées à cette nouvelle orthodoxie (Benko et al, 1996) expliquent le succès de ces régions par le rôle des variables endogènes ou les caractéristiques propres aux systèmes de production locaux et sur leurs capacités à s'adapter, à se renouveler et à innover (Fergène, 2013). Les premiers travaux sur la Troisième Italie mettaient tout d'abord l'accent sur les facteurs socioculturels à l'origine de cette vitalité (Bagnasco, 1977, Brusco, 1982) avant que Beccatini (1979) voit dans ce type d'organisation industrielle l'actualisation du district industriel de Marshall avec une forme de coordination hybride associant le marché et la réciprocité, ce que Bagnasco et Trigilia (1993) appellent la construction sociale $d u$ marché. Cette atmosphère industrielle est au cœur des économies externes d'agglomération sur lesquelles repose l'efficacité des entreprises - de petites de taille - du district. En France, ces travaux inspirent les recherches de Courlet et Pecqueur $(1991,1992)$ qui substituent à la notion du district celle du «système productif localisé » comme forme généralisée d'organisation productive territorialisée (Courlet, 2001) et censée mieux traduire l'ancrage territorial des processus économiques en œuvre et leur osmose avec la socio-culture locale (Fergène, 2013). De l'autre côté de l'Atlantique, les chefs de file de l'Ecole californienne de géographie économique Scott et Storper (1986) ont repris ces analyses pour expliquer les dynamiques de localisations industrielles dans les villes américaines. Mais contrairement à Beccatini qui exclue de ses travaux les 
districts situés dans les zones urbaines et ceux structurés par les grandes firmes, eux, plus fidèles à la conception marshallienne du district, s'intéressaient à des métropoles et mégalopoles où les avantages d'une industrie localisée se conjuguent avec ceux d'un marché du travail vaste et varié (Pecqueur et Rousier, 1992). Parallèlement à ces approches d'inspiration marshallienne, des approches en termes de districts technologiques (Antonnelli, 1986), de milieux innovateurs (Aydalot, 1986; Maillat et Perrin, 1992) et de région apprenante (Florida, 1995) s'inspirant des théories évolutionnistes, considèrent le territoire lui-même comme incubateur de technologie, de l'innovation, de connaissances et de nouvelles compétences. Cette conception territorialisée de l'innovation est le produit da la fécondation de la rencontre entre l'analyse de l'économie industrielle sur la diffusion des changements techniques et l'économie régionale. De nombreux travaux ont repris ensuite le concept du district sous des terminologies sémantiquement voisines telles que: Clusters, pôles de compétitivité, technopôles, grappe industrielle et filière que l'on désigne sous une appellation générique de modèles territoriaux d'innovation (TIMs).

Cette approche appréhende l'espace de façon plus large en faisant appel à des facteurs qui ne sont pas nécessairement économiques, mais qui relèvent aussi des structures socioculturelles, de l'organisation économique et politique, de la religion, de l'histoire et de la géographie.

Il en résulte que ce dernier n'est pas un support neutre, "il se transforme, s'adapte, se construit, se détruit par des acteurs et des facteurs multiples que l'on tente de classer en endogènes et exogènes, naturels, artificiels, virtuels, individuels et collectifs " (Lacour, 2009, p. 291). Son introduction dans l'analyse économique « $n^{\prime}$ apporte pas des raffinements de détail : elle change tout »(Ponsard, 1988b, p. 8).

\section{Conclusion : les défis de l'économie territoriale, ou comment construire une économie territoriale durable?}

Nous avons cherché à comprendre les raisons de l'ignorance puis de l'intégration lente et tardive de l'espace dans la pensée économique ainsi que les différentes manières dont celui-ci est appréhendé depuis les préclassique à nos jours. Il s'avère que la résistance de l'espace, dans sa dimension holistique, à toute modélisation mathématique est la raison principale d'une conception a-spatiale de l'économie par l'école marginaliste ; elle explique encore aujourd'hui l'ignorance par la Nouvelle économie géographique de ce que Marshal appelle l'atmosphère industrielle.

Si les préclassiques ont le mérite d'englobaient largement dans leurs réflexions la dimension spatiale de l'économie, depuis le début du XIXe siècle jusqu'au début des années 1980, les réflexions sur l'espace, développées par la théorie économique spatiale, la théorie de l'échange international et la science économique régionale avaient pour points communs d'aborder ce dernier sous l'angle fonctionnel, 
privilégiant une fonction ou un agent économique dominant (Courlet, 2008). Toutefois, la problématique n'est pas posée dans les mêmes termes, ainsi plusieurs approches de l'espace sont formulées: l'espace homogène comme l'espace de l'échange pour l'économie spatiale, l'espace polarisé comme l'espace de la firme motrice pour F. Perroux, l'espace centre-périphérie comme l'espace de la division internationale du travail pour les néomarxistes, l'espace de l'économie géographique comme l'espace des forces d'agglomération pour la Nouvelle économie géographique (Courlet, 2008).

Il fallait attendre le retournement des hiérarchies spatiales des années 1970 pour voir dans la notion d'espace celle du territoire, c'est-à-dire un milieu sociospatial structurant des comportements spatiaux, de l'organisation industrielle et de l'innovation.

Par sa capacité à intégrer et à valoriser les facteurs intangibles et non marchands et plus largement ce que Polanyi appelle l'économie plurielle, l'économie territoriale a su et pu s'imposer dans une conjoncture de relative stabilité économique. L'approfondissement de la mondialisation au cours de ces deux dernières décennies, le développement des nouvelles technologies de l'information et de la communication, l'accentuation de la crise écologique et des inégalités, dessinent une nouvelle conjoncture. L'approche territoriale du développement parviendraitelle à garder sa pertinence dans ce nouveau contexte ou serait-elle, elle aussi, dépassée comme ce fut le cas des approches citées dans ce texte?

Pour Rallet $(2006)^{8}$, l'idée même que l'économie locale fasse système est contestable. La transformation des économies actuelles fait que les cohérences économiques s'établissent de moins en moins au niveau local. En effet, comme le constatent Colletis-Wahl et al (2008) et Crévoisier (2010), le découplage entre lieu de production et lieu de consommation résultant de l'accélération des mobilités, réduit le rôle des activités induites dans les économies locales; suite à la croissance de la capitalisation boursière, les entreprises les plus déterminantes au niveau des territoires sont aujourd'hui massivement intégrées dans des groupes financiers; le développement et l'innovation se font tirer de plus en plus par des facteurs non technologiques, etc. Par ailleurs, la façon dont est théorisée l'innovation, c'est à dire en termes de l'éclosion de l'innovation technique répondant largement au crédo du marché (Moulaert et Sekia, 2003) conduit les modèles territoriaux d'innovation à l'ignorance ou à une prise en charge minimaliste des effets négatifs de leurs actions sur les hommes et la nature (Bernardy, 1999).

\footnotetext{
8 Commentaire de l'article «Economie régionale, économie territoriale : la dynamique des milieux innovateurs » de Crevoisier (2006), In Mollard et al (dir.), Territoires et enjeux du développement régional, Quae, pp.61-82.
} 
Si l'économie territoriale a su tenir compte de l'espace et du temps, son défit analytique majeur est à présent, l'intégration dans son projet de renouvellement de ses fondements théoriques et épistémologiques de ces questionnements multiples et surtout d'ordre éthique et morale comme l'environnement, la solidarité et l'équité.

Bâtir une théorie du développement territorial durable passe par l'articulation de deux approches que Planque et Gaussier (1999) qualifient de mésologiques - la problématique des modèles territoriaux d'innovation et la problématique $\mathrm{du}$ développement durable, susceptibles de comprendre le fonctionnement des systèmes complexes. Ces auteurs soutiennent que l'accent met par ces approches sur les interactions entre sous-systèmes de nature diverses conduit à la notion d'externalité comme élément clé de compréhension - dans une perspective analytique pour les modèles territoriaux d'innovation -et/ou de régulation - dans une perspective normative pour l'approche du développement durable - des écosystèmes humains et donc de leur adaptativité.

\section{Déclaration de divulgation}

Aucun conflit d'intérêts potentiel n'a été signalé par les auteurs.

\section{References}

Antonnelli, C. (1986). Technological Districts and Regional Innovation capacity. Revue d'économie régionale et urbaine, $\mathrm{n} 5$, pp. 695-705.

Aydalot, Ph. ed. (1986). Milieux innovateurs en Europe. Paris: GREMI, p. 361.

Aydalot, Ph. (1985). Economie Régionale et Urbaine. Paris: Economica, p. 487.

Aydalot, Ph. ed. (1984). Crise et espace. Paris : Economica, p. 350.

Bagnasco, A., Trigilia, C. (1993). La construction sociale du marché. Le défi de la troisième Italie. Cachan : ENS Cachan, p. 284.

Bagnasco, A. (1977). Tre Italie. La problematica territoriale dellosviluppo italiano. Bologna, il Mulino.

Bailly, A. (2009). La science régionale en perspective. Géographie, Economie, Société, 1/2009. Vol. 11, pp. 1-7.

Becattini, G. (1979). Dal "settore" industriale al "distretto" industriale. Alcuneconsiderazionisull'unità d'indaginedell'economiaindustriale. Rivista di economia e politicaindustriale, a. V, n. 1, pp. 721.

Benko, G. (2008). La géographie économique : un siècle d'histoire. Annales de Géographie, vol. 6, n 664, pp. 23-49.

Benko, G. (1999a). Entretien avec Alain Lipietz. Géographie, Économie et Société, Volume 1, n¹, pp. 217-232.

Benko, G. (1999b). Entretien avec Paul Krugman. Géographie, Économie et Société, Volume 1, n², pp. 403-423.

Benko, G. (1998). La science régionale. Puf, Paris, p. 126. 
Benko, G., Dunford, M., Lipietz, A. (1996). Les districts industriels revisités, In Pecqueur B (dir.), Dynamiques territoriales et mutations économiques. Paris : L'Harmattan, pp. 119-134.

Benko, G., Lipietz, A. (1992). Le nouveau débat régional : positions. In Benko, G. \&Lipietz, A. (dir.), Les régions qui gagnent, districts et réseaux: les nouveaux paradigmes de la géographie économique. Paris : Puf, pp. 13-32.

Bernardy, M. (1999). Technopoles: innovation tous azimuts et/ou intégration de l'environnement, In Benhayoun, G., Gaussier, N., \& Planque, B. (dir.), L'ancrage territorial du développement durable. Paris : L'Harmattan, pp. 83-103.

Borts, G., Stein, J. (1964). Economic growth in a free market. New York: Columbia University Press.

Bourdeau-Lepage, L., Huriot, J.-M. (2009). L'idée de région et le fait urbain. Revue d'Economie Régionale et Urbaine, n 2, pp. 267-288.

Brusco, S. (1982). The Emilian model: Productive decentralisation and social integration. Journal of Economics, Cambridge, Vol 6, n 2, pp. 164-184.

Camagni, R. (2004). Préface au livre de Matteaccioli A, In Philippe Aydalot pionnier de l'économie territoriale. Paris : L'Harmattan, pp. 13-17.

Camagni, R. (1996). Principes et modèles de l'économie urbaine. Paris: Economica, p. 382.

Capello, R. (2009). Regional growth and local development theories: conceptual evolution over fifty years of regional science. Géographie, économie, société, vol. 11, n 1, pp. 9-21.

Christaller, W. (1966). The Central places in Southern Germany, Prentice-Hall, Englewood, NJ.

Coissard, S. (2007). La nouvelle géographie économique de Paul Krugman, apports et limites. Revue $\mathrm{d}^{\prime}$ Economie Régionale et Urbaine, $\mathrm{n}^{\circ}$ 1, pp. 111-125.

Colletis-Wahl, K., Corpataux, J., Crevoisier, O. Kebir, L., Pecqueur, B. (2008). The territorial economy: a general approach in order to understand and deal with globalisation, In Aranguren M-J, Iturrioz C, WILSON J-R. (dir.), Networks, Governance and Economic Development: Bridging Disciplinary Frontiers. Cheltenham: Edward Elgar.

Combes, P.-P., Mayer, T., Thisse, J.-F. (2006). Economie géographique, l'intégration des régions et des nations Paris: Economica, p. 397.

Courlet, C., Pecqueur, B. (2013). L'économie territoriale. Presses universitaires de Grenoble, p. 142.

Courlet, C. (2008). L'économie territoriale. Presse universitaire de Grenoble, p. 135.

Courlet, C. (2001). Territoires et régions, les grands oubliés du développement économiques. Paris: L'Harmattan, p. 133.

Courlet, C., Pecqueur, B. (1992). Les systèmes industriels localisés en France: un nouveau modèle de développement, In Benko $\mathrm{G}$ et Lipietz A (dir.), Les régions qui gagnent, districts et réseaux : les nouveaux paradigmes de la géographie économique. Paris: Puf, pp. 81-102

Courlet, C, Pecqueur, B. (1991). Systèmes locaux d'entreprises et externalités : un essai de typologie. Revue d'économie régionale et urbaine, 3/4, pp. 391-406.

Crevoisier, O. (2010). La pertinence de l'approche territoriale. Economie Régionale et Urbaine, n 5, pp. 969-985.

Derycke, P.-H., Huriot, J.-M. (1996). Microéconomie et espace : quelle intégration? Revue économique, vol. 47, n 2, pp. 187-192.

Derycke, P.-H. (1994). L'intégration de l'espace dans le champ économique, in Auray J-P., Bailly A., Derycke P-H et Huriot J-M (dir.), Encyclopédie d'économie spatiale: Conceptscomportements-organisations. Paris : Economica, pp. 19-24.

Dockès, P. (1969). L'espace dans la pensée économique du XVI au XVIII siècle. Paris: Flammarion, p. 461. Ferguène, A. (2013). Economie territoriale et développement local : Concepts et expériences. Campus ouvert, p. 178. 
Florida, R. (1995). Toward the learning region. Futures, Vol. 27, n 5, pp. 527-536.

Garner, G. (2001). Penser l'espace, territoire et espace dans la théorie économique en Allemagne, 17501820. Revue d'histoire moderne \& contemporaine, vol. 48, n. 2/3, pp. 25-50.

Géneau de Lamarlière, I.-G., \&Staszak, J.-F. (2000). Principes de géographie économique. Paris : Bréal (Grand Amphi Géographie), p. 448.

Gérard-Varet, L.-A., Thisse, J.-F. (1997). Economie publique locale et économie géographique. Annales d'Economie et de Statistique, n. 45, pp. 1-35.

Hoyt, H. (1954). Homer Hoyt on Development of Economic Base Concept. Land Economics, vol. 30, n 2, pp. 182-186.

Heckscher, E. (1919). The effect of foreign trade on the distribution of income. In Heckscher-Ohlin trade theory. Cambridge, MA: MIT Press.

Hotelling, H. (1929). Stability in competition. The Economic Journal, vol 39, n 153, pp. 41-57.

Isard, W. (1956). Location and Space Economy. A General Theory Relating to Industrial Location, Market Areas, Land Use, Trade, and Urban Structure. Cambridge: MIT Press, p. 350.

Jacobs, J. (1984). Cities and the wealth of nations. New York: Random House.

Krugman, P. (1991). Increasing Returns and Economic Geography. The Journal of Political Economy, Vol. 99, No. 3, pp. 483-499.

Lacour, C. (2009). Une nouvelle science régionale?. Revue d'Economie Régionale et Urbaine, n 2, pp. 289-314.

Lösch, A. (1954). The economics of location. Yale University Press, p. 520.

Maillat, D., \& Perrin, J.-C. (1992). Entreprises innovatrices et développement régional. Neuchâtel: GREMI-EDES.

Martin, R., Sunley, P. (2000). L'économie géographique de Paul Krugman et ses conséquences pour la théorie du développement régional: une évaluation critique, In Benko, G., \& Lipietz, A. (dir.), Larichesse des régions, la nouvelle géographie socio-économique. Paris: Puf, pp. 33-84.

Martin, R. (1999). The New Geographical Turn in economics: Some Critical Reflections. Cambridge Journal Economics, Volume 23, n 1, pp. 65-91.

Moulaert, F., Sekia, F. (2003). Territorial Innovation Models. Regional Studies, vol. 37, n3, pp. 289-302.

North, D. (1955). Location Theory and Regional Economic Growth. The Journal of Political Economy, vol. 63, n. 3, pp. 243-258.

Nussbaumer, J. (2002). Le rôle de la culture et des institutions dans le débat sur le développement local : la contribution de l'école historique allemande, thèse d'économie, Université des Sciences et Technologies de Lille.

Ohlin, B. (1933). Interregional and international trade. Cambridge: Harvard University Press.

Pecqueur, B., Rousier, N. (1992). Les districts technologiques, un nouveau concept pour l'étude des relations technologies-territoires. Canadian Journal of Regional Science, XV, 3, pp. 437-455.

Perrin, J.-C. (1992). Pour une révision de la science régionale. L'approche par les milieux. Canadian Journal of Regional Science, XV.2, pp. 155-197.

Planque, B., Gaussier, N. (1999). Milieux innovateurs et développement durable, des mésologies complémentaires? In Benhayoun G, Gaussier N Planque B (dir.), L'ancrage territorial du développement durable. Paris : L'Harmattan, pp. 273-286.

Polanyi, K. (2009). La Grande transformation: aux origines politiques et économiques de notre temps. Paris: Gallimard, p. 467.

Ponsard, C. (1988a). Les espaces économiques flous, In Ponsard, C. (dir.), Analyse économique spatiale, Paris: Puf, pp. 355-390. 
Ponsard, C. (1988b). Introduction, In Ponsard, C. (dir.), Analyse économique spatiale, Paris: Puf, pp. 721.

Ponsard, C. (1975). Préface au livre de Mougeot M, Théorie et politique économiques régionales. Paris: Economica, pp. 1-13.

Ponsard, C. (1955). Economie et espace: Essai d'intégration du facteur spatial dans l'analyse économique. Rennes: Imprimerie Oberthur, p. 468.

Scott, A.J., Storper, M. (1986). Production, Work, Territory: The Geographical Anatomy of Industrial Capitalism. Boston, Allen and Unwin.

Thisse, J.-F. (2007). Nouvelle économie géographique et géographie : quel dialogue?. Espace géographique 3, pp. 193-214.

Thisse, J.-F. (1997). L'oubli de l'espace dans la pensée économique. Revue Région et Développement, $\mathrm{n}$ 6, pp. 1-29.

Thisse, J.-F. (1992). Espace et concurrence : une cohabitation difficile? In Derycke, P.-H. (dir.) Espace et dynamiques territoriales. Paris: Economica, pp. 113-136.

Walras, L. (1874). Eléments d'économie politique pure, ou, théorie de la richesse sociale. Lausanne, p. 407.

Weber, A. (1909). Theory of the location of industries. Chicago: The University of Chicago Press, p. 256. 